\title{
O CUIDADO DE ENFERMAGEM AO PACIENTE ONCOLÓGICO FORA DE POSSIBILIDADE DE CURA: PERCEPÇÃO DE UM GRUPO DE PROFISSIONAIS
}

\author{
Maria Helena Pinto' , Maria Fernanda Cruz², Claudia Bernardi Cesarino' ${ }^{1}$, Adriana Pelegrini dos Santos Pereira ${ }^{3}$, \\ Rita de Cássia Helu Mendonça Ribeiro ${ }^{4}$, Lúcia Marinilza Beccaria ${ }^{1}$
}

\begin{abstract}
RESUMO: Esta pesquisa investigou a experiência do profissional da enfermagem no cuidado ao paciente oncológico fora de possibilidade de cura; trata-se de um estudo de abordagem qualitativa, com participação de 13 profissionais da equipe de enfermagem de um hospital de ensino do interior paulista. Os dados foram coletados entre agosto e outubro/2009, por meio de entrevista. A análise de conteúdo de Bardin possibilitou a categorização dos dados em: O cotidiano da enfermagem com o paciente oncológico fora de possibilidade de cura; $\mathrm{O}$ enfrentamento do trabalho com paciente em fase terminal e Dificuldades no trabalho com o paciente fora de possibilidade de cura. Os resultados apontaram que a assistência aos pacientes restringiu-se aos cuidados físicos e o enfrentamento se deu com estratégias individuais, podendo colocar em risco a segurança do paciente. $\mathrm{O}$ despreparo dos profissionais para o cuidado emocional foi a principal dificuldade relatada, com evidências de necessidade de sua capacitação. PALAVRAS-CHAVE: Enfermagem; Câncer; Paciente terminal; Pessoal de saúde.
\end{abstract}

\section{NURSING CARE FOR PATIENTS WITH INCURABLE CANCER: THE PERCEPTION OF A GROUP OF HEALTHCARE PROFESSIONALS}

\begin{abstract}
This study investigated the experience of professional nurses involved in the care of cancer patients whose cancer was beyond treatment. The study had a qualitative approach and was undertaken with the participation of thirteen professionals from a nursing team in a teaching hospital in the countryside in Sao Paulo state. The data was collected through interviews between August and October 2009. By applying Bardin's content analysis, the data was categorized in three groups; nursing routines with patients with incurable cancer, facing work with patients in the terminal phase, and difficulties in working with patients beyond cure. The results indicated that patient care was limited to physical care, and that facing work with these patients was accomplished with individual strategies, putting patient safety at risk. The health care professionals' lack of preparedness for emotional care was the main difficulty related, with evidence for the need for training in this matter. KEYWORDS: Nursing; Cancer; Terminally ill patients; Health professionals.

\section{EL CUIDADO DE ENFERMERÍA AL PACIENTE ONCOLÓGICO SIN POSIBILIDAD DE CURA: PERCEPCIÓN DE UN GRUPO DE PROFESIONALES}

RESUMEN: Esta investigación analizó la experiencia del profesional de enfermería en el cuidado al paciente oncológico sin posibilidad de cura. Se trata de un estudio de abordaje cualitativo, con participación de 13 profesionales del equipo de enfermería de un hospital de enseñanza del interior paulista. Los datos fueron recogidos entre agosto y octubre/2009, por medio de entrevista. El análisis de contenido de Bardin posibilitó la categorización de los datos en: El cotidiano de la enfermería con el paciente oncológico sin posibilidad de cura; El afrontamiento del trabajo con paciente en fase terminal; y Dificultades en el trabajo con el paciente sin posibilidad de cura. Los resultados apuntaron que la asistencia a los pacientes se restringió a los cuidados físicos y el afrontamiento se dió con estrategias individuales, siendo posible poner en riesgo la seguridad del paciente. La falta de preparación de los profesionales para el cuidado emocional fue la principal dificultad relatada, con evidencias de necesidad de su capacitación. PALABRAS CLAVE: Enfermería; Cáncer; Paciente terminal; Personal de salud.

\footnotetext{
${ }^{1}$ Enfermeira. Doutora em Enfermagem. Professora do Departamento de Enfermagem Geral da Faculdade de Medicina de São José do Rio Preto - FAMERP.

${ }^{2}$ Enfermeira.

${ }^{3}$ Enfermeira. Mestre em Enfermagem Fundamental. Doutoranda em Ciências da Saúde pela FAMERP. Professora do Departamento de Enfermagem Geral da FAMERP.

${ }^{4}$ Enfermeira. Doutora em Ciências da Saúde. Professora do Curso de Graduação e Coordenadora do Curso de Especialização em Enfermagem em Nefrologia da FAMERP.
} 


\section{INTRODUÇÃO}

Atualmente, os avanços tecnológicos nas biociências têm favorecido a sobrevivência dos pacientes com câncer, e com isso há um aumento do número de internações hospitalares de pacientes com câncer em fase terminal, fora de possibilidade de cura. No Brasil, o câncer é a segunda causa de morte na população, representando quase $17 \%$ dos óbitos de causa conhecida ${ }^{(1)}$. Devido ao despreparo da família e sofrimento do paciente diante da dor intensa, fadiga e outros sintomas angustiantes, estas mortes acabam acontecendo no hospital.

O paciente fora de possibilidade de cura exige da equipe de profissionais, principalmente da Enfermagem, não só habilidade técnica para realizar cuidados físicos, mas habilidade para o cuidado emocional pautado na ética e na humanização.

Na experiência durante o ensino clínico do curso de graduação, na assitência com os pacientes oncológicos fora de possibilidades terapêutica, percebeu-se um distanciamento entre profissional e paciente, certa frieza no cuidado, possivelmente por não saber lidar com o paciente nesta condição clínica. Conviver com o processo de morte é um contraste com o curar e "salvar vidas" aprendido durante a formação biomédica tradicional destes profissionais $^{(2-3)}$. Estar ao lado do paciente terminal pode fazer o profissional da saúde lembrar-se da sua própria finitude e da fragilidade humana diante das adversidades da $v \operatorname{vida}^{(4)}$. Aceitar o fato de que a vida tem prazo de validade desconhecido é o que torna a morte uma questão difícil de ser discutida, enfrentada e pesquisada ${ }^{(5)}$.

Estudos têm constatado que a morte é vista como inimiga, sinônimo de fracasso; provoca sentimentos de culpa, tristeza e impotência, o que muitas vezes faz com que o profissional da saúde utilize mecanismos de defesa como distanciamento do doente, atitudes automatizadas e contenção dos próprios sentimentos ${ }^{(6)}$. $\mathrm{O}$ enfrentamento da morte depende de fatores sociais, culturais e espirituais, assim como da qualidade dos cuidados que, muitas vezes, está relacionada aos custos econômicos, acessibilidade e comunicação paciente-equipe de saúde ${ }^{(7)}$.

Um estudo realizado no Chile, com pessoas muito próximas das falecidas, demonstrou que a satisfação dos pacientes com os cuidados prestados, a comunicação, a frequência de atenção e bom humor do profissional são identificados como fatores que interferem na qualidade do processo de morrer, independente do morrer em casa ou no hospital, posto que o desejo do paciente de morrer em casa, muitas vezes, não pode ser atendido por falta de disponibilidade de recursos humanos e físicos adequados ${ }^{(7-8)}$.
Em outro estudo, o autor destaca que não é a própria morte que desperta temor e terror, mas a imagem antecipada da morte, que hoje em dia vem revestida de isolamento, sofrimento e dor ${ }^{(9)}$. Estes temas suscitam debates e dilemas nos quais não se deixa de inovar na abordagem de aspectos de humanização e a defesa de uma "ética da vida"(3).

Considerando que o profissional da enfermagem é aquele que permanece a maior parte do tempo ao lado do paciente e que, assim como outros profissionais da saúde, foi preparado para curar, promover a longevidade e, de certa forma, ignorar o fenômeno da morte como se fosse algo que não fizesse parte da vida, temse a questão: Como é o trabalho com o paciente com câncer em fase terminal, fora de possibilidade de cura, para os profissionais da enfermagem?

Para discutir o tema em questão é importante lembrar que o princípio do cuidado ao paciente fora de possibilidade terapêutica deve pressupor cuidados nas dimensões física, psicológica e socioespiritual. A preservação da qualidade de vida do paciente ganha prioridade sobre a preservação da vida, com valorização do cuidado emocional, traduzido em empatia, manifesta pelo saber ouvir, tocar e falar olhando nos olhos do paciente, com o objetivo de promover conforto e bem-estar do paciente e família até os momentos finais da vida do doente ${ }^{(10)}$. Esta modalidade de cuidado é denominada de cuidados paliativos ${ }^{(11)}$.

Os cuidados paliativos não se caracterizam por apressar ou postergar a morte com práticas tecnologizadas, tratamentos caros e invasivos, mas de favorecer o alívio da dor e de outros sintomas angustiantes, oferecendo apoio à família durante o processo de doença e processo de morrer do paciente, com o objetivo de garantir qualidade de vida a ambos ${ }^{(4)}$. As ações de enfermagem valorizam o sofrimento e as conquistas do paciente, o que fortalece o ser cuidado e quem cuida, ajudando-os a encontrar significados para as situações vivenciadas ${ }^{(10)}$.

Hoje, os cuidados paliativos constituem um desafio para as instituições e profissionais da saúde. Estes cuidados devem ser prestados por uma equipe multiprofissional, com objetivos bem definidos e com competência para atender as reais necessidades, quer sejam físicas, afetivas, emocionais, psicológicas ou espirituais da unidade paciente/família ${ }^{(4)}$.

Assim, o preparo do profissional para o cuidado paliativo é uma necessidade emergente na área da saúde e, como ponto de partida, este estudo buscou compreender a experiência do profissional da enfermagem no cuidado ao paciente oncológico, fora de possibilidade de cura, em um local onde esta situação é uma constante. 


\section{METODOLOGIA}

Trata-se de um estudo de abordagem qualitativa no qual o estudo de caso com vários sujeitos foi a estratégia metodológica selecionada, por possibilitar conhecer a individualidade no modo de ver e enfrentar as diversidades vividas, além de possibilitar maior compreensão dos significados atribuídos pelos profissionais de enfermagem na condição de trabalho com pacientes oncológicos em fase terminal.

O estudo foi desenvolvido em um hospital de ensino do interior do Estado de São Paulo. Os pacientes da oncologia, nesta instituição, são acompanhados pelas várias especialidades médicas, nas diversas unidades de internação, enquanto que os pacientes que se apresentam em fase terminal ficam, de preferência, em uma unidade constituída de dezesseis leitos, divididos em três ambientes; a escala de serviço mantém um auxiliar ou técnico em enfermagem para cada três ou quatro leitos, e um enfermeiro em cada plantão.

O tempo de internação dos pacientes nesta unidade é, em média, de dez dias, e os óbitos chegam a 20 por mês; as visitas aos pacientes são permitidas por uma hora, diariamente.

Participaram do estudo 13 profissionais da equipe de enfermagem que, após convite, manifestaram o desejo de ser informantes da pesquisa. Foram incluídos no estudo os profissionais com atividades há mais de um ano na unidade, nos turnos manhã ou tarde. Foram excluídos aqueles que desenvolviam suas funções no período noturno e os que se encontravam afastados das atividades durante o período de coleta dos dados.

As informações foram obtidas por meio de uma entrevista, seguindo um roteiro com dados de caracterização dos participantes e com questões norteadoras: Fale um pouco sobre o seu trabalho com pacientes oncológicos em fase terminal; O que você pensa sobre a morte; Quais as dificuldades enfrentadas no seu trabalho. As entrevistas foram realizadas no local de trabalho, em sala reservada, com os profissionais que se colocaram disponíveis para responder as questões, no período de agosto a outubro de 2009. Cada entrevista teve, em média, a duração de 20 minutos; com um lápis e papel, a pesquisadora registrou as respostas dos informantes, os quais confirmaram a veracidade do conteúdo descrito após o término da entrevista.

Para análise dos dados, optou-se pela técnica de análise de conteúdo proposta por Bardin ${ }^{(12)}$, com o intuito de descobrir o significado do conteúdo manifesto, seguindo as fases de pré-análise, exploração dos dados e tratamento e interpretação dos resultados. Para tanto, os relatos foram submetidos a leituras sucessivas, estabelecendo uma codificação denominada de unidade de significado. Estas foram analisadas à luz das indagações propostas, que convergiram em três categorias temáticas.

As categorias temáticas evidenciam o que foi expresso nos relatos, possibilitando a compreensão dos significados apreendidos acerca da experiência dos profissionais da enfermagem no trabalho com pacientes oncológicos, fora de possibilidade de cura.

O projeto da pesquisa foi aprovado pelo Comitê de Ética em Pesquisa da Faculdade de Medicina de São José do Rio Preto, protocolo n. 3516/2009, e os participantes assinaram oo Termo de Consentimento Livre e Esclarecido, atendendo as regras da Resolução CNS 196/96.

Os participantes foram identificados com a letra "E", seguida do número de ordem da sequência dos entrevistados, para garantir o anonimato.

\section{RESULTADOS}

Participaram do estudo dez auxiliares de enfermagem e três enfermeiros, sendo um do sexo masculino, com idades entre 25 e 41 anos, e com tempo de trabalho na unidade variando de 13 meses a 12 anos. A partir dos relatos dos entrevistados, apreendeu-se que a experiência dos profissionais da enfermagem com pacientes oncológicos, em fase terminal, é rotineira e difícil pelas constantes mortes; o dia a dia de trabalho acontece com ações restritas a cuidados físicos e pouco envolvimento com os pacientes.

Assim, os dados desta pesquisa estão expressos em três categorias: $O$ cotidiano da enfermagem com o paciente oncológico fora de possibilidade de cura; $O$ enfrentamento do trabalho com pacientes em fase terminal e Dificuldades no trabalho com o paciente fora de possibilidade de cura.

\section{$O$ cotidiano da enfermagem com o paciente fora de possibilidade de cura}

Para o profissional da enfermagem, as atividades realizadas com pacientes em fase terminal não são diferentes daquelas realizadas com outro tipo de paciente; são rotineiras, resumidas em cuidados físicos diretos e observação, sem destaque para o cuidado emocional ou psicológico, como observado nos relatos de E3, E9 e E17 que têm como exemplo o descrito a seguir: 
Não há diferença; é medicar, verificar sinais vitais, higiene, dieta e observação clínica [...]. (E1)

Da mesma maneira, nos relatos de E4, E8, E10 e E13, semelhantes à fala:

Fazer higiene intima, banho, colocar sentado, fazer balanço hídrico, curativo das úlceras, mudar de decúbito. (E2)

O cuidado emocional à família também foi lembrado pelos informantes, embora sejam reconhecidos a dificuldade e o despreparo destes profissionais:

Dar suporte emocional para familia também é esperado do profissional, mas é difícil! Nem sempre isto é possivel. [...] Eu não sei muito bem o que fazer. (E9)

Os participantes relataram sentimentos ou experiências diárias com o paciente fora de possibilidade terapêutica como:

[...] ansiedade, apreensão, expectativa de melhora da dor e frustração com o não-alcance do objetivo [...]. Depois do efeito do analgésico a dor volta [...] é como se nadasse, nadasse e morresse na praia. É desanimador! (E7)

Para os profissionais, a morte do paciente com câncer avançado alivia o sofrimento do doente e família conforme falas de E11 e E10, bem como nos seguintes relatos:

A morte é o fim de um sofrimento para quem está com câncer. (E5)

Para os pacientes terminais, eu vejo que a morte é o alivio para a dor. Um descanso. (E7)

Tanto para o paciente como para a família, a morte significa alivio. (E6)

\section{$O$ enfrentamento do trabalho com o paciente em fase terminal}

O enfrentamento do trabalho com pacientes fora de possibilidade de cura e com a proximidade do fim da vida é uma dificuldade verbalizada pelos profissionais:

O cuidado, neste momento da vida, não é só prestar o cuidado para o alivio da dor, mas tratar a mente, pois é difícil enfrentar [...] saber que vai morrer. Apesar da experiência, jamais consegui entender os mecanismos da vida e da morte. (E4)

A espiritualidade, de alguma forma, está presente no enfrentamento do trabalho pelos profissionais:

Procuro encarar o trabalho de uma forma natural. Aqui é aqui; em casa é outra coisa. (E11)

Quando saio daqui, desligo absolutamente de tudo. (E3)

Faço leitura de livros espíritas, orações. Eu acredito na vida após a morte. (E4)

Eu tenho muita fé. Acredito que Deus dá força e que as coisas acontecem, porque têm que acontecer. Eu estou aqui, não é por acaso. (E12)

Estranhamente, uma das estratégias mencionadas para enfrentar o dia a dia de trabalho com paciente fora de possibilidade de cura está apresentada no relato:

Eu deixo o rádio ligado com música. Assim, não presto atenção nos gemidos de dor dos pacientes. (E2)

\section{Dificuldades no trabalho com o paciente fora de possibilidade de cura}

A principal dificuldade relatada no trabalho com pacientes oncológicos, fora de possibilidade terapêutica, foi a falta de preparo profissional, o que pode ter influência direta no desempenho de suas atividades junto ao paciente:

Nem todos os funcionários são iguais [...]. É importante fazer que todos entendam que os cuidados devem ser prestados até o fim da vida, e conscientizar a equipe de saúde que isso não é investir no paciente, e sim garantir uma morte digna. O que não é fácil! Nós não fomos preparados para lidar com a morte, mas com vida e cura. (E5)

A estrutura física e o horário de visita aos pacientes também foram lembrados como uma das dificuldades vividas pelos profissionais da enfermagem:

Gostaria que tivesse, na unidade, um local especifico, com espaço suficiente para o paciente ficar junto com 
a família, com música ambiente, paredes coloridas, para que assim o paciente tenha uma atenção especial e uma morte com dignidade. (E7)

Penso que os pacientes deveriam ficar acompanhados por um familiar e não ter apenas uma hora de visita por dia, como é a norma. (E6)

\section{DISCUSSÃO}

Aceitar o diagnóstico de uma doença fora de possibilidade de cura é uma situação complexa para o ser humano, e entender o significado que as pessoas atribuem à doença, morte e cuidado é uma forma de respeitar a cultura de cada indivíduo no planejamento da assistência à saúde individualizada. Acredita-se que o conceito elaborado acerca da morte pelos profissionais, somado à forma de interação da equipe durante o processo de decisão sobre o cuidado de pacientes em fase terminal, pode direcionar o cotidiano de trabalho com pacientes e familiares no final da vida do doente; além de dar sentido à vida dos profissionais e mobilizar energias positivas para a melhora da qualidade de vida dos pacientes e de sua própria vida ${ }^{(13)}$.

Os cuidados prestados ao paciente fora de possibilidade de cura, para a Organização Mundial de Saúde (OMS), visam a promoção de conforto e bem-estar, e são basicamente voltados para higiene, alimentação, curativos e cuidados gerais, assim como para a atenção à analgesia, para o alívio do sofrimento ou dor, a estes se acrescenta o cuidado emocional e espiritual que implica em amparar, apoiar e confortar a unidade paciente/família desde a descoberta do câncer avançado, tornando o profissional ainda mais presente no processo de terminalidade e depois, durante o período de luto ${ }^{(11)}$. O alívio da dor é, atualmente, visto como um direito humano básico e, portanto, trata-se não apenas de uma questão clínica, mas também de uma situação ética e humanizada que envolve o trabalho de todos os profissionais de saúde ${ }^{(14)}$.

A analgesia objetiva o alívio da dor, mas este cuidado pode ser acrescido de outras atitudes que permeiam a relação humana, como o contato humano e apoio psicológico, fazendo com que a dor que não se solucione com medicamentos seja amenizada ou controlada com atitudes de empatia ${ }^{(15)}$. Durante a interação com os pacientes ou familiares é importante saber escutar ativamente, observar a linguagem corporal, ouvir não só o que as pessoas estão dizendo, mas também como estão dizendo. Dessa maneira, é possível reconhecer as reais necessidades dos pacientes e familiares e cuidar de forma integral ${ }^{(16)}$. Para tanto, o adequado preparo dos profissionais é estratégia fundamental para o controle da dor e sintomas prevalentes em pacientes com câncer em fase avançada, tendo em vista a responsabilidade do enfermeiro na avaliação das condições gerais do paciente e o planejamento da assistência a ser prestada.

As intervenções de enfermagem, principalmente na situação de cuidados a pacientes fora de possibilidade terapêutica, devem ser traçadas objetivando proporcionar uma experiência menos dolorosa também à família, com cuidado especial na transmissão da notícia da morte e no preparo do ambiente, garantindo a privacidade e respeito ao tempo necessário para a despedida do doente ${ }^{(17)}$.

Quando se trata de paciente oncológico em fase avançada, o profissional enfrenta um grande desgaste emocional pelo contexto do trabalho, que envolve o controle da própria ansiedade e da depressão acompanhado do sofrimento e morte do ser cuidado ${ }^{(8)}$. Cuidar de pacientes fora de possibilidade de cura é vivenciar e compartilhar momentos de amor e compaixão, aprendendo que é possível proporcionar o morrer com dignidade; é garantir aos pacientes a certeza de não estarem sozinhos no momento da morte; é oferecer cuidado integral e atenção humanística associada à batalha do controle dos sintomas; é contribuir para que a sociedade perceba que é possível desassociar a morte e o morrer do medo e da dor ${ }^{(11)}$.

A filosofia de cuidados paliativos, segundo a OMS, visa a avaliação correta e a identificação precoce dos problemas de ordem física, psicossocial e espiritual, sempre com origem nas necessidades individuais de conforto, bem-estar, e prevenção da dor, além de afirmar a vida e encarar o morrer como um processo natural, promovendo um sistema de apoio para o paciente viver ativamente tanto quanto possível até a morte ${ }^{(12)}$.

A formação do profissional de enfermagem traz uma pré-concepção em relação ao ser humano, com experiências sobre o processo de morte e morrer que variam com as circunstâncias que rodeiam a morte, o tipo de relação entre o doente e o profissional e a qualidade de mecanismos de enfrentamento ${ }^{(9)}$.

O câncer ainda é entendido pelas pessoas, em geral, como sinônimo de dor, morte e sofrimento. Nesta perspectiva, cabe ao profissional identificar suas próprias concepções relativas ao câncer e morte e estabelecer estratégias de enfrentamento, visando uma assistência adequada e eficaz que possibilite minimizar o sofrimento de todos os envolvidos no processo do cuidar e do morrer ${ }^{(15)}$. Os momentos terminais que são vivenciados por esses pacientes expõem dilemas humanos e os modos como cada um trata a própria vida. 
Os profissionais no dia a dia com o paciente fora de possibilidade terapêutica mencionaram, algumas vezes, estratégias de enfrentamento que não são comuns. Considerando a perspectiva psicossocial, o ser humano tem capacidade de responder de forma consistente aos desafios e dificuldades, de reagir com flexibilidade e capacidade de superação diante de circunstâncias desfavoráveis, como o contato estreito com a terminalidade ou finitude da vida, dor, desesperança, e o desgaste constante e presente como no cotidiano do trabalho com o paciente com câncer fora possibilidade terapêutica ${ }^{(11)}$.

As relações interpessoais representam a principal dificuldade, não só da equipe de enfermagem, como de todos os profissionais da saúde no ambiente conturbado, devido à demanda excessiva de pacientes, limitação de estrutura física e solicitações de pacientes e familiares carentes de informações. No contexto de cuidados paliativos, a comunicação verbal e não verbal é considerada fundamental para as relações terapêuticas entre paciente, família e profissional de saúde ${ }^{(18)}$.

A espiritualidade está relacionada a um conjunto de valores íntimos que dá sentido à vida, e dessa maneira produz capacidade de suportar sentimentos como frustração, raiva e ansiedade, o que pode melhorar a qualidade de vida das pessoas ${ }^{(14)}$. A fé, a oração e a crença numa vida depois da morte são fontes de apoio para alguns profissionais da área de saúde lidarem com as preocupações espirituais e com o sofrimento de pacientes no final da vida ${ }^{(19)}$.

O relato de ligar o rádio para não ouvir as queixas do paciente fere os princípios bioéticos e humanísticos das ciências da saúde. A música, quando posta a trabalho da melhoria das atividades humanas não deve ser ouvida, mas apenas percebida. Os ritmos devem chegar ao subconsciente e criar uma sensação de bem-estar para eliminar a tensão de espírito. Em regra, utiliza-se a música durante períodos breves, de até duas horas e meia, devendo-se evitar períodos prolongados ${ }^{(20)}$; não deve desviar a atenção do ambiente de trabalho, onde o foco são as necessidades do paciente. A utilização da música de forma inadequada pode comprometer a segurança do doente; a falta de identificação de responsabilidades profissionais favorece as ocorrências indesejadas.

A experiência do câncer é um processo que desafia a pessoa e, para tanto, os demais que convivem de alguma maneira com os doentes buscam algo com o qual possam enfrentar esses desafios. Algumas atitudes refletem as dificuldades enfrentadas no processo de trabalho diário da enfermagem com paciente fora de possibilidade de cura.

Uma das dificuldades mencionadas pelos infor- mantes foi a deficiência na formação profissional sobre questões de morte e o próprio morrer. Este tema tem sido destaque em alguns estudos, com evidências de despreparo e inadequação do pessoal diante de situações que envolvem morte. Isto, muitas vezes, faz com que o profissional mantenha certo distanciamento do paciente, como estratégia de proteger-se do próprio sofrimento ${ }^{(6)}$.

O investimento na formação profissional em cuidados paliativos pode proporcionar menor sofrimento a todos os envolvidos, incluindo o paciente e familiar, além de minimizar o custo do cuidado ao sistema de saúde e melhorar a qualidade dos cuidados durante o processo de morrer ${ }^{(8)}$.

Para os pacientes em cuidados paliativos preconiza-se ficar em local que permita a permanência de familiares, para que estes também se sintam acolhidos, com acesso às informações médicas sobre mudanças no quadro clínico e etapas do processo de morrer. O ambiente deve prover necessária privacidade para que questões íntimas possam ser conversadas e para que o momento da morte possa ser respeitado como único e individualizado. Paciente e familiar devem receber atendimento das suas necessidades por parte dos membros da equipe de saúde ${ }^{(4)}$.

\section{CONSIDERAÇÕES FINAIS}

No contexto estudado, apreendeu-se que os profissionais entendem que o trabalho com pacientes oncológicos, fora de possibilidade de cura, é mais que cuidar do físico. No entanto, eles não estão preparados para o cuidado emocional. A estratégia utilizada para sobreviver no dia a dia de trabalho nem sempre visa segurança do paciente, sendo mencionadas como dificuldades a estrutura física inadequada para o convívio do paciente com a família.

Compreender a experiência dos profissionais no trabalho com pacientes em fase terminal implica em contribuir para mudanças no preparo desse profissional, disponibilizando-o mais para a interação com o paciente e família. Por outro lado, o trabalho com estes pacientes é estressante e o profissional também precisa de suporte para lidar com o processo de morte e morrer com responsabilidade. Cabe ao enfermeiro, como coordenador da equipe de enfermagem, em conjunto com a equipe interdisciplinar, incluir momentos de reuniões com o grupo de profissionais para que estes possam também ser cuidados, com discussões sobre conflitos vividos no dia a dia do trabalho. Isto poderá melhorar sobremaneira a qualidade da assistência aos pacientes fora de possibilidade de cura e o bem-estar dos profissionais.

Este estudo é apenas um ponto de partida para 
reflexões sobre as experiências vivenciadas por estes profissionais, dado que o tema é complexo, envolve formação, crenças e experiências individuais que transformam a visão de mundo ao longo da vida.

\section{REFERÊNCIAS}

1. Ministério da Saúde (BR). Instituto Nacional do Câncer. Estimativa 2010 - Incidência de câncer no Brasil. Rio de Janeiro; 2010. [acesso em 2010 ago 10] Disponível: http://wwwl.inca.gov.br/estimativa/2010/.

2. Rodrigues MVC, Ferreira ED, Menezes TMO. Comunicação da enfermeira com pacientes portadores de câncer fora de possibilidade de cura. Rev Enferm UERJ. 2010;18(1):86-91.

3. Silva CHD, Schramm FR. Bioética da obstinação terapêutica no emprego da hemodiálise em pacientes portadoras de câncer do colo do útero invasor, em fase de insuficiência renal crônica agudizada. Rev Bras Cancerol. 2007;53(1):17-27.

4. Moraes TM. Como cuidar de um doente em fase avançada de doença. O Mundo da Saúde. 2009;33(2):231-8.

5. Silva KS, Ribeiro RG, Kruse MHL. Discursos de enfermeiras sobre morte e morrer: vontade ou verdade? Rev Bras Enferm. 2009;62(3):451-7.

6. Ruedel LM, Beck CLC, Silva RM, Lisboa RL, Prochnow A, Prestes FC. Relações interpessoais entre profissionais de enfermageme familiares em unidade de tratamento intensivo: estudo bibliográfico. Cogitare Enferm. 2010;15(1):147-52 .

7. Santos MCL, Pagliuca LMF, Fernandes AFC. Cuidados paliativos al portador de cáncer: reflexiones según la visión de Paterson y Zderad. Rev Latino-Am Enfermagem. 2007;15(2):350-4

8. Venegas ME, Alvarado OS. Fatores relacionados à qualidade do processo de morrer na pessoa com câncer. Rev Latino-Am Enfermagem. 2010;18(4):725-31.

9. Bifulco VA; Iochida LC. A formação na graduação dos profissionais de saúde e a educação para o cuidado de pacientes fora de recursos terapêuticos de cura. [acesso em 2011 set 01]. Disponível: http:// www.scielo.br/ Rev Bras Educ Med. 2009;33(1)

10. Pimenta CAM. Cuidados paliativos: uma nova especialidade do trabalho da enfermagem? Acta Paul Enferm. 2010;23(3):vii-xii.
11. Nader Marta GN, Hanna SA, Silva JLF. Cuidados paliativos e ortotanásia. Diagn Tratamento. 2010;15(2):58-60.

12. Bardin L. Análise de conteúdo. $20^{\mathrm{a}}$ ed. Lisboa: Edições 70; 2009.

13. Guimarães HP, Avezum A. O impacto da espiritualidade na saúde física. Rev Psiquiatr Clín. (São Paulo). 2007;Suppl 1:88-94.

14. Ministério da Saúde (BR). Instituto Nacional de CâncerINCA. Ações de enfermagem para o controle do câncer: uma proposta de integração ensino-serviço. Rio de Janeiro: Ministério da Saúde/ Instituto Nacional de Câncer; 2008.

15. Stumm EMF, Leite MT, Maschio G. Vivências de uma equipe de enfermagem no cuidado a pacientes com câncer. Cogitare Enferm. 2008;13(1):75-82.

16. Allen S, Crouch A. Cultural and spiritual health assessment. Vital Notes for Nurses: Health Assessment. 2005;10:311-30.

17. Poles K, Bousso RS. Compartilhando o processo de morte com a família: a experiência da enfermeira na UTI pediátrica. Rev Latino-Am Enfermagem. 2006;14(2):207-13.

18. Oliveira AC, Silva MJP. Autonomia em cuidados paliativos: conceitos e percepções de uma equipe de saúde. Acta Paul Enferm. 2010;23(2):212-7.

19. Martinez Quês AA, Montoro CH, González MG. Fortalezas e ameaças em torno da segurança do paciente segundo a opinião dos profissionais de enfermagem. Rev Latino-Am Enfermagem. 2010;18(3):339-45.

20. Gatti MFZ, Silva MJP. Ambient music in the emergency services: the professionals' perception. Rev Latino-Am Enfermagem. 2007;5(3):377-83. 